RESEARCH REPORT

\title{
DETERMINING THE POTENCY OF CARDIAC REHABILITATION PHASE- II IN CVD PATIENTS WITH CO-MORBIDITIES
}

\begin{abstract}
Globally, cardiovascular disease is a leading cause of mortality and it is estimated that CVD is a cause of approximately $29.6 \%$ of all the deaths worldwide. According to a research published in 2015 in American journal of Cardiology it is a time to "rebrand and reinvigorate" the cardiac rehabilitation program as the existing program appears to be neither financially practicable nor maintainable mainly due to affordability barriers. A Quasi experimental study was conducted on 40 participants whom were diagnosed with cardiovascular disease with comorbidities (diabetes mellitus and dyslipidemia). Participants were asked to enroll in phase-ll cardiac rehabilitation program on the recommendation of the cardiologist and were given intervention based on the guideline of American College of Sports medicine. Six weeks of supervised exercise protocol was conducted and its dosage was measured via Frequency, Intensity, Time and Type (FITT) protocol. While for determining the potency of phase-II Cardiac Rehabilitation program assessment of the outcome measures were made after every sessions and the results obtained from the assessment had revealed that for a CVD patients with diabetes 24 hours of exercise which would be performed for not less than four weeks provides a maximal result which carried on for further two weeks whereas for CVD patients with Dyslipidemia 20 hours of exercise had provided a desired result carried on for the remaining weeks. The study concluded that exercises based cardiac rehabilitation had a significant impact in improving the patient condition where a six week of training protocol improves the outcome measures included in the study.
\end{abstract}

\section{KEYWORDS}

CVD, Potency of Exercise, Dyslipidemia, Diabetes Mellitus, ACSM, $A B I, 6 M W T, W I Q$

\author{
Sumaira Imran Farooqui \\ Associate Professor/Principal \\ Ziauddin College of Rehabilitation Sciences \\ Ziauddin University \\ sumairaimranfarooqui@gmail.com

\section{Ali Farhad} \\ Assistant Professor \\ Ziauddin College of Rehabilitation Sciences \\ Ziauddin University \\ alifarhadtharwani@gmail.com

\section{Muhammad Usman Khan} \\ Assistant Professor \\ Ziauddin College of Rehabilitation Sciences \\ Ziauddin University \\ m_khanusman@hotmail.com
}

[Farooqui SI, Khan MU, Farhad A. Determining the Potency of Cardiac Rehabilitation Phase- II in CVD Patients with Co-morbidities. Pak. j. rehabil. 2018;7(1):15-20] 


\section{INTRODUCTION}

Globally, cardiovascular disease is a leading cause of mortality and it is estimated that CVD is a cause of approximately $29.6 \%$ of all the deaths worldwide'. Cardivascular diseases is a group of disease that includes both Coronary artery disease and coronary heart disease ${ }^{2}$. The prevalence and the incidence of the disease increased with age and has an adverse overall prognosis with $45 \%$ of mortality rate after diagnosis within 5 years ${ }^{3}$. Although the incidence of the disease decreased over time in developed countries, indeed still it is a leading cause of deaths among adults in low and middle income countries ${ }^{4}$. With the advancement in the health care system during the last two decades the live expectancy of the people around the globe has been increased ${ }^{5}$ along with the increase in the number of people living with symptomatic coronary


ed by European Society of Cardiology in 2016, management of cardiovascular disease can be achieved by incorporating basic life style modification like physical activity, cessation of smoking and healthy diet? ${ }^{7}$. According to American College of Cardiology, 2016 exercise based rehabilitation program provides a multi-dimensional interventional strategy towards improving the prognosis of $C V D^{8}$. The importance of exercise based interventional strategies for cardio vascular fitness can be estimated from the fact that it's efficacy has been established well over 25 years ago", and now the time has arrived where the researcher should look towards introducing an exercises based approach which can full fill the demands of those patients whom due to some reasons like socioeconomics conditions, poor health care facilities and huge financial effects unable to complete recommended supervised exercises seesions ${ }^{10}$. Recently numbers of international societies on cardiology like European Society of Cardiology, American college of cardiology, World Health Organization etc. has emphasized on cost effectiveness of prevention strategies for cardiovascular disease and recommended for measures aimed to promote healthy life style changes at the general population level ${ }^{11}$. The existing protocol of cardiovascular rehabilitation as per the guideline of American college of Sports Medicine comprises of four phases where phase II which is also called as the transitional phase comprises of 10 to 12 weeks of supervised exercises sessions which mainly emphasized on functional improvement and patient education ${ }^{12}$. This phase has extreme importance because it is based on risk stratification and life style modification ${ }^{13}$.

Cardiac Rehabilitation as evident from multiple previous studies is effective in reducing mortality rate of approximately $20 \%{ }^{14}$ still the enrollment rate in the program is very low which is mainly because of huge financial burden incurred by enrolling into the program ${ }^{15}$. Other factors that causes low rate of enrollment includes lack of unified method for referral, communication gap among rehabilitation teams, patients and Cardiac rehabilitation facilities, lack of awareness related to cardiac rehabilitation among the population and lastly limited access of patients to cardiac rehabilitation facilities. As highlighted in the American Heart Association science recommendations efforts must be made to systematically increase participation in Cardiac Rehabilitation by: 1) escalating research based cardiac rehabilitation program 2) patient and family education 3) Educating health care providers 4) reducing specific barriers to referral and participation in cardiac rehabilitation that attributes to patients, physician and community 5) promoting better understanding of cardiac rehabilitation program 6) making cardiac rehabilitation program cost and time effective 7) expanding the spectrum of responsibilities of physical therapist, nurses, physician and families of patients 8) introducing a concept of rehabilitation teams in hospitals and health care setups 9) increasing the effectiveness of the program through introducing the concept of secondary prevention treatment option and the management of the chronic disease and lastly 10) elimination of a general concept that Cardiac rehabilitation program is less effective in comparison to pharmacological management of cardiac problem. Thus making cardiac rehabilitation as an essential part of Cardio Vascular Disease management and prevention (primary and Secondary). According to a research published in 2015 in American journal of Cardiology it is a time to "rebrand and reinvigorate" the cardiac rehabilitation program as the existing program appears to be neither financially practicable nor maintainable mainly due to affordability barriers ${ }^{16}$. This provides for introducing a tailored physical therapy rehabilitation program which should comprise of practicing skills and activities that enable patients to utilize their general or strength endurance in motor activities, aerobic interval and endurance training, strength endurance training (inspiratory muscle training and peripheral muscle training), practicing functions activities, and training to reduce cardiovascular risk factors. Hence the present study is to identify the potency of phase-II cardiac rehabilitation program on cardiovascular disease patients with different comorbidities.

\section{METHODOLOGY}

A Quasi experimental study was conducted on 40 participants, diagnosed with cardiovascular disease with comorbidities (diabetes mellitus and dyslipidemia). Participants were asked to enroll in phase-II cardiac rehabilitation program on the recommendation of the cardiologist and were given intervention based on the guideline of American College of Sports medicine ${ }^{17}$. Six weeks of 
supervised exercise protocol was conducted and its dosage was measured via Frequency, Intensity, Time and Type (FITT) protocol. Frequency of exercise was 5 days/week; Intensity was measured by using Karvonean formula initially the intensity was kept at $40 \%$ of MHR which was gradually increase upto $70 \%$ depending on the ability of patients to perform, time was 40- 50 minutes per session and type of exercise includes treadmill and cycling. For the purpose of determining the potency of exercise program each session of exercise was estimated to be equal to one hour and the outcome measures were estimated before and after every session of exercise for the period of six weeks of conditioning. The patients were divided into two groups on the basis of their comorbidities. Patients with CVD and Diabetes mellitus were recruited in group $A$ and CVD with dyslipidemia were recruited in group $B$. The interventional strategies for both the group were same and the outcome measures were recorded via Ankle Brachial Index ${ }^{18}$, Six Minute Walk Test $(6 \mathrm{MWT})^{19}$ and Walking Impairment Questionnaire $(W I Q)^{20}$. To effectively calculate the impact of prescribed exercises regime daily exercise session time was notice and the outcome measures were taken before and after every exercise session. All the participants were asked to complete the 6 weeks of exercise protocol.

\section{Exercise Termination Criteria}

The exercise session was prematurely been terminated on the existence of one of the following conditions.

- Blood saturation level less than 90;

- Borg rate of perceived exertion>7;

- Any sign of Arrhythmia;

- Bradicardia;

- Tachycardia and

- On the request of the participants

\section{Inclusion and Exclusion Criteria \\ Inclusion Criteria}

- Age 18-75 years ${ }^{21}$

- Left ventricular ejection fraction >3021

- Coronary Vascular Disease $22-23$

- Ability to perform Exercise ${ }^{22-23}$

\section{Exclusion Criteriaa ${ }^{21}$}

- Uncontrolled Atrial or Ventricular tachyarrhythmia

- Atrial Fibrillation

- Moderate to Severe Valvular Disease

- Pericarditis

- Anemia

- Renal Disease

- Cor-pulmonale

- COPD

\section{Ethical Considerations ${ }^{24}$}

Ethical considerations were made according to guidelines provided under Belmont report for human subjects. The data provided by the participants were kept confidential; consent was taken prior to the recruitment of the participants and was given opportunity to ask any question before, during and after the completion of the study.

\section{RESULTS}

The conditioning was done for a period of six weeks according to the guideline provided by American College of Sports Medicine (ACSM). The demographic information shows that the mean age of the participants was 60 years whereas the gender wise distribution shows more male participants than female as shown in figure.1



To exclude the effects of age related biasness on the outcome measure the participants were evaluated on the basis of their age. The age wise distribution of the participants was shown in figure 2.

The effects of phase II cardiac rehabilitation significantly improves the outcome measures, Ankle Brachial Index of the participants improves in both

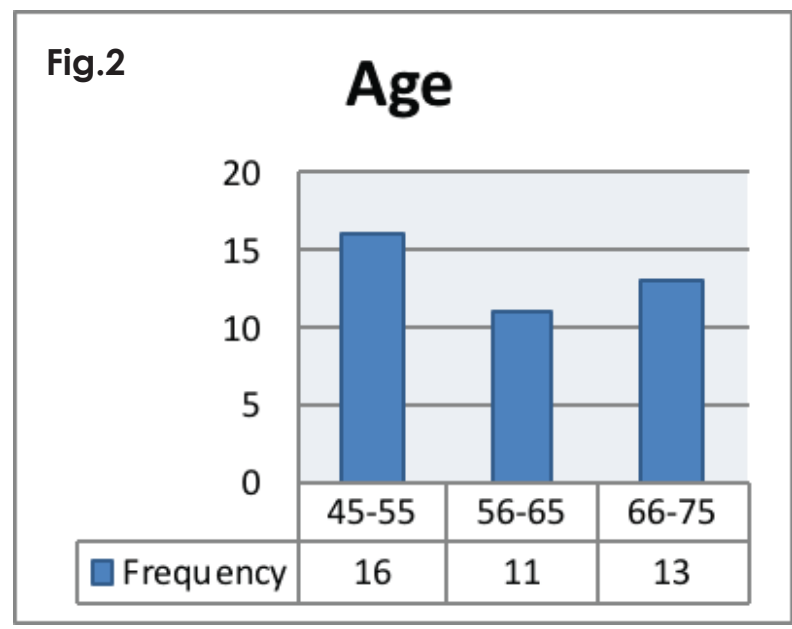

the group $p<0.05$.The mean $A B I$ of the participants in Group A and B was 0.7 and 0.84 which improved to 1.04 and 1.05 respectively. (Fig. 3) 


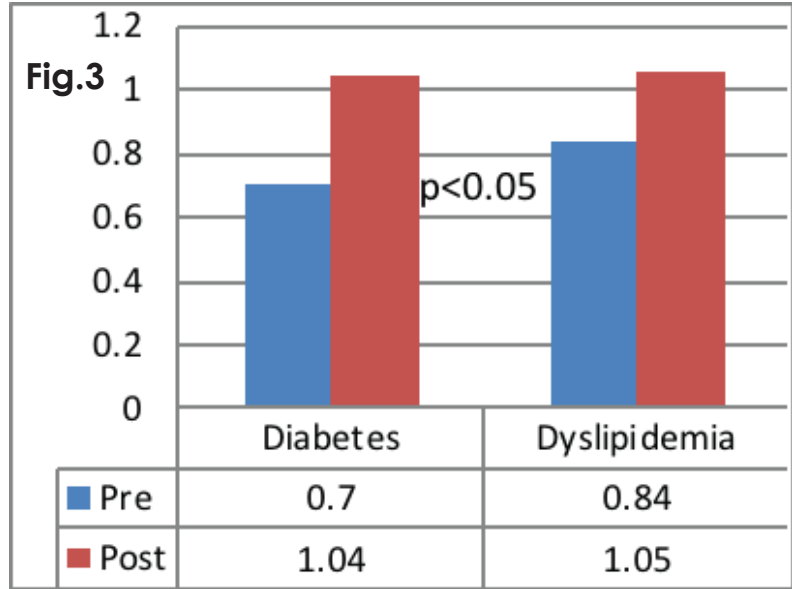

Similar effects were also noticed on Six Minute walk test and Walking impairment questionnaire where conditioning of six weeks had provided significant in improving the distance covered by the participants during six minute walk test and on walking impairment questionnaire $\mathrm{p}<0.05$. (Figure 4)

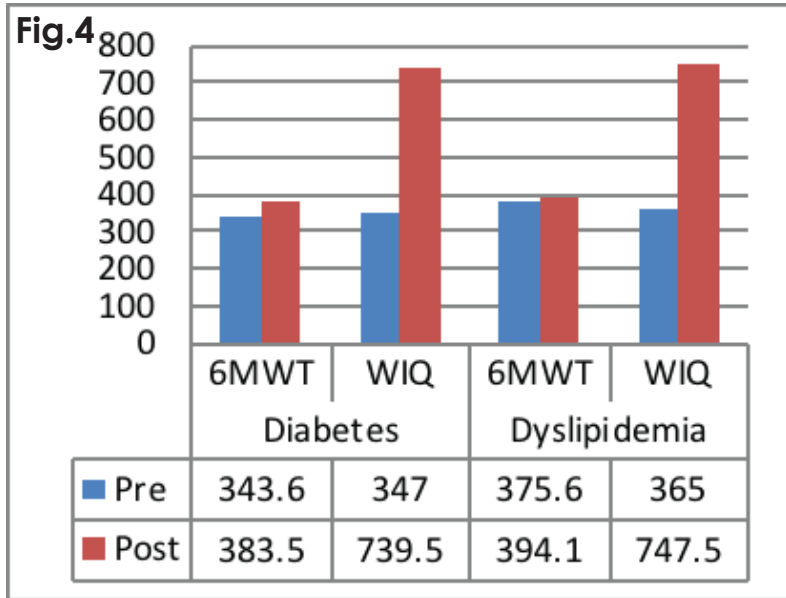

Table 1-*WIQ scores were multiplied by 10 for the purpose of better understanding for figure 4

To identify the impact of age on the outcome measures Pearson coefficient of correlation was determined which revealed no relationship between

\begin{tabular}{|c|c|c|c|c|c|c|}
\hline \multirow[b]{2}{*}{$\begin{array}{l}\text { Hours } \\
\text { of } \\
\text { Exercise }\end{array}$} & \multicolumn{3}{|c|}{ Diabetes } & \multicolumn{3}{|c|}{ Dyslipidemia } \\
\hline & $A B I$ & 6MWT & WIQ & ABI & $6 \mathrm{MWT}$ & WIQ \\
\hline 3 & 0.7 & 343.6 & 34.7 & 0.84 & 375.6 & 36.5 \\
\hline 6 & 0.7 & 346.9 & 40.5 & 0.96 & 378 & 35 \\
\hline 9 & 0.8 & 350.8 & 45 & 1 & 380 & 40 \\
\hline 12 & 0.9 & 360 & 49 & 1.02 & 385.5 & 44.5 \\
\hline 15 & 1 & 375.8 & 55.5 & 1.02 & 388 & 50 \\
\hline 18 & 1.02 & 380.2 & 60.8 & 1.04 & 390 & 60.85 \\
\hline 21 & 1.03 & 383.5 & 70.8 & 1.05 & 394.1 & 75 \\
\hline 24 & 1.04 & 383.5 & 73.95 & 1.05 & 394.1 & 75 \\
\hline 27 & 1.04 & 383.5 & 73.95 & 1.05 & 394.1 & 75 \\
\hline 30 & 1.04 & 383.5 & 73.95 & 1.05 & 394.1 & 75 \\
\hline
\end{tabular}

age and the outcome measures i.e. $A B I, 6 M W T$ and $W I Q(R=0.12,0.49 \& 0.028$ respectively) which further confirm that age of the participants had no direct impact on the outcome measures use in this study. Moreover it was also revealed that no relationship was exist between 6MWT and $W I Q(R=0.15)$ which may be due to the fact that WIQ was subjective approach of assessment whereas 6MWT had objective approach.

While for determining the potency of phase-II Cardiac Rehabilitation program assessment of the outcome measures were made after every sessions and the results obtained from the assessment had revealed that for a CVD patients with diabetes 24 hours of exercise which would be performed for not less than four weeks provides a maximal result which carried on for further two weeks whereas for CVD patients with Dyslipidemia 20 hours of exercise had provided a desired result which also carried on for the remaining weeks. The hour wise description was illustrated in Table 1

The hour wise distribution of the outcome measures as shown in Table 1 revealed that the impact of phase-II cardiac rehabilitation was more prompt in cardiovascular disease patients having Dyslipidemia as comorbidity than from among the patients having diabetes mellitus. The result obtained from the study clearly identifies that among dyslipidemia patient the maximum improvement in all the three outcome measures i.e. Ankle Brachial Index (ABI), Six Minute Walk Test (6MWT) and Walking Impairment Questionnaire (WIQ) were obtained after 18 hours of exercise (3rd week of rehabilitation session) whereas among the group of patients having diabetes with Cardiovascular disease the maximum impact on the outcome measures were achieved after 21 hours of exercise which make it a 4th week of rehabilitation session. Moreover the result obtained from the study also revealed that a conditioning of six weeks of exercise session did not improve outcome measure any further once the maximum effects of the exercises were achieved.

\section{DISCUSSION}

The result provided significant evidence in favor of Phase-II cardiac rehabilitation program where the conditioning exercises based on the guidelines of American College of Sports medicine improved the outcome measure of the participants in both the groups. For the purpose of quantifying the dosage of exercise in both the group Frequency, Intensity, Time and Type protocol was used. The demographic details had revealed that more male were enrolled in the rehabilitation program than females, because females may due to extensive training program and lack of transportation facilities available for them were less interested in enrolling themselves. Moreover it was also noticed that female 
may not consider rehabilitation worth important to get themselves enrolled the findings was in accordance to the study conducted by Pattanshetty et al in 2016 who highlighted that $72.78 \%$ of the females diagnosed with cardiovascular disease were not attending the cardiac rehabilitation program for which inconvenient location, followed by financial burden and transportation difficulty were the top three barriers identified by them from among the list of eleven different barriers ${ }^{12}$. The same study had also revealed that $23.85 \%$ of the participants believed that the program was too much expensive to be worth enrolling, making it a second most barrier after inconvenient location (34.4\%). The result of the study conducted by Achttien et al 2015 had provided result in favor of the present study, according to Achttien exercise based cardiac rehabilitation improves endurance and health related quality of life among heart failure patients ${ }^{25}$. Moreover, it was also highlighted that cardiac rehabilitation program should be designed according to the need based approach in which the preference must be given to the patients limitations established during the assessment procedure. Sandesera at al conducted a study in 2015 had also concluded the similar findings regarding the current exercises base rehabilitation program which was turned out to be less effective in term of patient priority because of the barriers like financial feasibility, duration and limited referrals making the program less viable and sustainable in the long run and recommended to 'rebrand and reinvigorate' the training program that would be financially feasible, cost-effective and to be designed according to the need of the individual $^{16}$. Guidelines of the European Society of Cardiology had also emphasized on designing an exercise protocol that would be according to the need of individual and should met the requirement of the patients need in term of financial viability ${ }^{2}$. Keeping on record the recommendations and the guidelines provided by different international organization the present study was designed to determine the cutoff point on which the maximal benefit was attained so that the program could be tailored up to the level where the patients get maximal benefit within the limited duration. The study provide a significant evidence in favor of different international guidelines which had greatly emphasized on the patient need approach for cardiac rehabilitation. However, study had some limitations, the study was first of its type in Pakistan where adequate facilities for cardiac rehabilitation were not readily available for all and it was therefore a sample size of only 40 patients were collected, moreover due to poor health care facilities and lack of infrastructure the data was collected only from single tertiary care hospital and no account were given to the patients enrolled in different other tertiary care hospitals of the city. Lastly, the pool effects of the outcome measures were analyzed and the effects of training session on individual bases were not discussed in detail. Hence, more researches were required in this regards with large sample size and from the people of different striate of the society so that a conclusive proof can be obtained regarding the tailoring of the exercise program according to individuals need.

\section{CONCLUSION}

The study concluded that exercises based cardiac rehabilitation had a significant impact in improving the patient condition where a six week of training protocol improves the outcome measures included in the study. However, according to the recent guidelines of different international societies which has emphasized on tailoring the cardiac rehab program according to the needs of patients so that maximum number of people can be brought within the sphere of rehabilitation the current study was designed and the potency of cardiac rehabilitation phase II was determined and it was found that 20-25 hours of supervised exercise program which should be performed in not less than four weeks provides a maximal effects that could be achieved after 12 weeks of recommended program.

\section{REFERENCES}

[1] Donnino MW, Andersen LW, Berg KM, Reynolds JC, Nolan JP, Morley PT, Lang E, Cocchi MN, Xanthos T, Callaway CW, Soar J. Temperature management after cardiac arrest: an advisory statement by the advanced life support task force of the International Liaison Committee on Resuscitation and the American Heart Association Emergency Cardiovascular Care Committee and the Council on Cardiopulmonary, Critical Care, Perioperative and Resuscitation. Resuscitation. 2016:98:97-104.

[2] Piepoli MF, Hoes AW, Agewall S, Albus C, Brotons C, Catapano AL, Cooney MT, Corrà U, Cosyns B, Deaton C, Graham I. ESC Scientific Document Group. 2016 European guidelines on cardiovascular disease prevention in clinical practice: the Sixth Joint Task Force of the European Society of Cardiology and other societies on cardiovascular disease prevention in clinical practice. Eur Heart J. 2016;37(29):2315-81.

[3] Oliveira GB, Avezum A, Roever L. Cardiovascular disease burden: evolving knowledge of risk factors in myocardial infarction and stroke through population-based research and perspectives in global prevention. Front Cardiovasc Med. 2015 Aug 13;2:32.

[4] Mozaffarian D, Benjamin EJ, Go AS, Arnett DK, Blaha MJ, Cushman M, Das SR, De Ferranti S, Després JP, Fullerton HJ, Howard VJ. Executive summary: heart disease and stroke statistics-2016 update: a report from the American Heart Association. Circulation. 2016;133(4):447-54. 
[5] Mathers CD, Stevens GA, Boerma T, White RA, Tobias MI. Causes of international increases in older age life expectancy. The Lancet. 2015; 385(9967):540-8.

[6] Chatterij S, Byles J, Cutler D, Seeman T, Verdes E. Health, functioning, and disability in older adults - present status and future implications. The Lancet. 2015;385(9967):563-75.

[7] Galiè N, Humbert M, Vachiery JL, Gibbs S, Lang I, Torbicki A, Simonneau G, Peacock A, Vonk Noordegraaf A, Beghetti M, Ghofrani A. 2015 ESC/ERS guidelines for the diagnosis and treatment of pulmonary hypertension: the Joint Task Force for the Diagnosis and Treatment of Pulmonary Hypertension of the European Society of Cardiology (ESC) and the European Respiratory Society (ERS): endorsed by: Association for European Paediatric and Congenital Cardiology (AEPC), International Society for Heart and Lung Transplantation (ISHLT). Eur. Heart J. 2015;37(1):67-119.

[8] Levine GN, Bates ER, Bittl JA, Brindis RG, Fihn SD, Fleisher LA, Granger CB, Lange RA, Mack MJ, Mauri L, Mehran R. 2016 ACC/AHA guideline focused update on duration of dual antiplatelet therapy in patients with coronary artery disease: a report of the American College of Cardiology/American Heart Association Task Force on Clinical Practice Guidelines. J Thorac Cardiovasc Surg . 2016;152(5):1243-75.

[9] Plach SK. Women and cardiac rehabilitation after heart surgery: patterns of referral and adherence. Rehabil Nurs. 2002;27(3):104-9.

[10] Daly J, Sindone AP, Thompson DR, Hancock K, Chang $E$, Davidson P. Barriers to participation in and adherence to cardiac rehabilitation programs: a critical literature review. Progress in cardiovascular nursing. 2002;17(1):8-17.

[11] Balady GJ, Ades PA, Bittner VA, Franklin BA, Gordon NF, Thomas RJ, Tomaselli GF, Yancy CW. American Heart Association Science Advisory and Coordinating Committee. Referral, enrollment, and delivery of cardiac rehabilitation/secondary prevention programs at clinical centers and beyond: a presidential advisory from the American Heart Association. Circulation. 2011; 124(25):2951-60.

[12] Pattanshetty RB, Chintamani R, Ribeiro S. PHASE ॥ CARDIAC REHABILITATION PROGRAM-BARRIERS TO FOLLOW UP AND PARTICIPATION: A CROSS-SECTIONAL STUDY. Int J Physiother Res. 2016;4(3):1489-97.

[13] Chintamani R, Ribeiro SG. Phase 2 cardiac rehabilitation program-barriers to Follow up and participation-a cross-sectional study.

[14] Joel H, Emily G, Mary A, Richard A, Melissa D. Depression and cardiac rehabilitation attendance by Medicare beneficiaries after hospitalization for myocardial infaction. J Cardiopulm Rehabil Prev. 2014;34:284.

[15] Balady GJ, Ades PA, Bittner VA, Franklin BA, Gordon NF, Thomas RJ, Tomaselli GF, Yancy CW. American Heart Association Science Advisory and Coordinating Committee. Referral, enrollment, and delivery of cardiac rehabilitation/secondary prevention programs at clinical centers and beyond: a presidential advisory from the American Heart Association. Circulation. 2011; 124(25):2951-60.

[16] Sandesara PB, Lambert CT, Gordon NF, Fletcher GF, Franklin BA, Wenger NK, Sperling L. Cardiac rehabilitation and risk reduction: time to "rebrand and reinvigorate". Journal of the American College of Cardiology. 2015;65(4):389-95.

[17] American College of Sports Medicine. ACSM's guidelines for exercise testing and prescription. Lippincott Williams \& Wilkins; 2013 Mar 4.

[18] Nishimura H, Miura T, Minamisawa M, Ueki Y, Abe N, Hashizume N, Mochidome T, Harada M, Shimizu K, Shoin W, Yoshie K. Prognostic value of ankle brachial index for future incident heart failure in patients without previous heart failure: data from the impressive predictive value of ankle brachial index for clinical long term outcome in patients with cardiovascular disease examined by $A B \mid$ study. Heart and vessels. 2017;32(3):295-302.

[19] Boucher C, Buchanan K. Early Utilization Of The 6-Minute Walk Test (6MWT) In An Active Patient After Cardiac Surgery-A Case Report.

[20] Murphy TP, Cutlip DE, Regensteiner JG, Mohler III ER, Cohen DJ, Reynolds MR, Massaro JM, Lewis BA, Cerezo J, Oldenburg NC, Thum CC. Supervised exercise, stent revascularization, or medical therapy for claudication due to aortoiliac peripheral artery disease: the CLEVER study. J Am Coll Cardiol. 2015;65(10):999-1009.

[21] Fontes-Carvalho R, Sampaio F, Teixeira M, Gama $\mathrm{V}$, Leite-Moreira AF. The role of a structured exercise training program on cardiac structure and function after acute myocardial infarction: study protocol for a randomized controlled trial. Trials. 2015;16(1):90.

[22] Salmoirago-Blotcher E, Wayne PM, Dunsiger S, Krol J, Breault C, Bock BC, Wu WC, Yeh GY. Tai chi is a promising exercise option for patients with coronary heart disease declining cardiac rehabilitation. J. Am. Heart Assoc. 2017;6(10):e006603.

[23] Blog RL. Focus on Fitness: Exercise and Heart Health-Rundown of the Latest Research By Jennifer Van Pelt, MA Today's Dietitian Vol. 20, No. 2, P. 52.

[24] Department of Health E. The Belmont Report. Ethical principles and guidelines for the protection of human subjects of research. The Journal of the American College of Dentists. 2014;81 (3):4. Achttien RJ, Staal JB, Van der Voort S, Kemps HM,

[25] Koers H, Jongert MW, Hendriks EJ, Practice Recommendations Development Group. Exercise-based cardiac rehabilitation in patients with coronary heart disease: a practice guideline. Neth Heart J. 2013;21 (10):429-38. 